\title{
Prävention und Früherkennung
}

\author{
Stefan Andreas ${ }^{a *}$ Fernando Gamarra $^{\mathrm{b} *}$ Felix Herth ${ }^{\mathrm{c} *}$ Hans Hoffmann ${ }^{\mathrm{d} *}$ Dennis Nowak ${ }^{\mathrm{e} *}$ \\ Susanne Riha ${ }^{\mathrm{f} *}$ Rainer Thomasius ${ }^{\mathrm{g} *}$ Dag Wormanns ${ }^{\mathrm{h} *}$ \\ a Lungenfachklinik Immenhausen, Kreis Kassel,

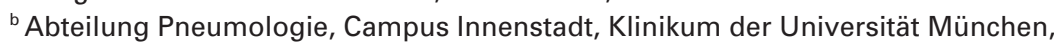 \\ ${ }^{c}$ Abteilung für Pneumologie und Beatmungsmedizin, Thoraxklinik am Universitätsklinikum Heidelberg, \\ dAbteilung für Thoraxchirurgie, Thoraxklinik am Universitätsklinikum Heidelberg, \\ e Institut und Poliklinik für Arbeits-, Sozial- und Umweltmedizin, Campus Innenstadt, Klinikum der Universität München, \\ ${ }^{f}$ Zentrum für Pneumologie, Allergologie, Beatmungsmedizin, Thorax- und Gefäßchirurgie, Krankenhaus Coswig, \\ ${ }^{g}$ Deutsches Zentrum für Suchtfragen des Kinder- und Jugendalters, Universitätsklinikum Hamburg-Eppendorf, \\ ${ }^{\mathrm{h}}$ Evangelische Lungenklinik Berlin, Deutschland
}

\section{Prävention und \\ Früherkennung aus arbeitsmedizinischer Sicht}

Etwa $80 \%$ der in Deutschland beruflich verursachten Krebserkrankungen sind Lungenkarzinome, die in $80 \%$ der Fälle auf eine Asbestexposition zurückgehen. An zweiter Stelle der häufigsten kanzerogenen Arbeitsstoffe steht ionisierende Strahlung (Radon-222), der vor allem die beim ostdeutschen Uranabbau eingesetzten Bergleute ausgesetzt waren [1]. Unter präventiven Aspekten bemerkenswert ist dabei, dass die große Mehrzahl der als Berufskrankheit anerkannten Krebserkrankungen der Lunge bei Rauchern auftreten; durch Verzicht auf Rauchen könnten vermutlich mehr berufsbedingte Karzinome verhindert werden als durch die Elimination sämtlicher kanzerogener Arbeitsstoffe [2].

Der Anteil an Lungenkarzinomen, die von den betroffenen Patienten beruflichen Noxen zugeschrieben werden, variierte in einer Auswertung von 32 internationalen Studien zwischen 0 und $40 \%$ [3]. Auch wenn berufsbedingte Karzinome nur einen Anteil von 5\% ausmachen, wären in Deutschland bei jährlich 420000 Krebsneuerkrankungen 21000 Fälle berufsbedingt. Tatsächlich werden jedes Jahr aber weniger als 6000 Krebserkrankungen mit Verdacht auf eine Berufskrankheit gemeldet. Dies deutet auf eine inakzeptabel hohe Dunkelziffer hin. Ursächlich hierfür sind in erster Linie fehlende Meldungen der behandelnden Ärzte; die teilweise restriktive Haltung einiger Unfallversicherungsträger ist quantitativ nachrangig.

*Alle Autoren haben zu gleichen Teilen zu diesem Artikel beigetragen.

\section{Prävention und Kompensation}

Im Hinblick auf erforderliche Präventionsmaßnahmen werden Arbeitsstoffe von unabhängigen Kommissionen, wie der International Agency for Research on Cancer (IARC) und der Senatskommission zur Prüfung gesundheitsschädlicher Arbeitsstoffe der Deutschen Forschungsgemeinschaft (DFG), nach ihrem kanzerogenen Potenzial bewertet. Die Klassifikation eines Arbeitsstoffs als nachgewiesenes Humankarzinogen (K1) korreliert bei beruflich durch entsprechende Exposition erworbenen Erkrankungen in den meisten Fällen mit der Aufnahme dieser Krankheiten in die Liste der Berufskrankheiten, die bei Vorliegen eines begründeten Verdachts gesetzlich meldepflichtig sind. Wenn eine berufliche Noxe wie beispielsweise Passivrauchen am Arbeitsplatz zwar als Humankanzerogen klassifiziert ist, aber noch keine entsprechende Berufskrankheit definiert ist, sollte (mit Einverständnis des betroffenen Patienten) eine Meldung nach der «Öffnungsklausel» erfolgen.

\section{Asbest: Primärprävention und Früherkennung}

Die positiven Auswirkungen eines konsequenten Verbots karzinogener Arbeitsstoffe auf das Lungenkarzinom-Risiko zeigen sich besonders deutlich bei Asbest. Allein durch die Tatsache, dass die Produktion und Verarbeitung von Asbest in Deutschland bereits 1993 und damit 12 Jahre vor einer entsprechenden EU-weiten Regelung verboten wurde, konnten in Deutschland mindestens 20000 Lungenkarzinome und Mesotheliome vermieden werden.

Die Früherkennung von Lungentumoren bei Personen mit Asbest-Exposition wurde unter anderem im Asbestos Surveillance Program Aachen untersucht. Von 5389 ehemaligen

\section{KARGER}

Fax +497614520714

Information@Karger.de

www.karger.com
(C) 2011 S. Karger GmbH, Freiburg

0378-584X/11/3416-0002\$38.00/0

Accessible online at:

www.karger.com/onk 


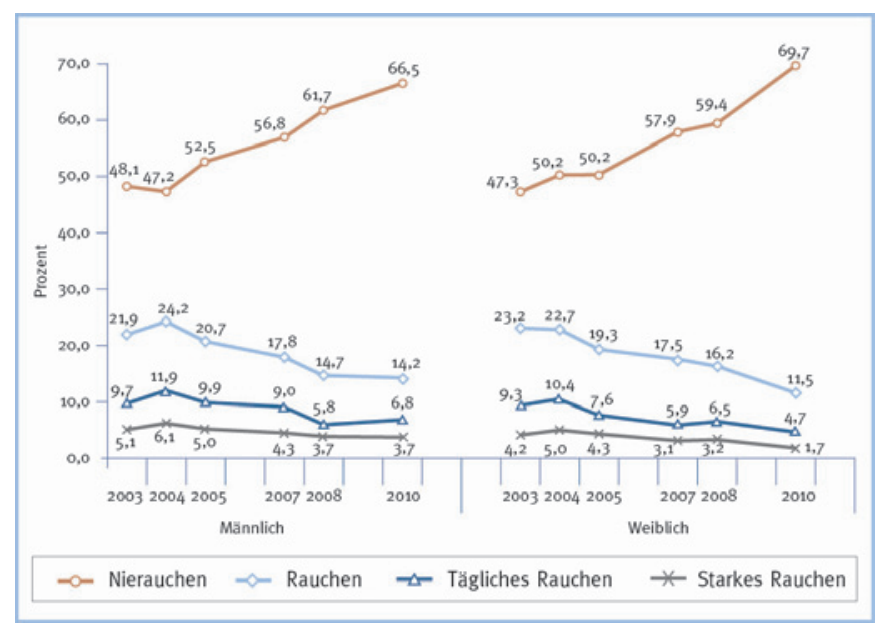

Abb. 1. BZgA-Drogenaffinitätsstudie: Rauchverhalten bei Kindern und Jugendlichen im Alter von 12-17 Jahren (modifiziert nach [7, 8]).

Kraftwerksarbeitern wurden 316 aufgrund der langjährigen Asbest-Exposition (durchschnittlich 29,7 Jahre), ihres Alters und Nikotinkonsums als Hochrisiko-Personen klassifiziert. Bei 187 in die Studie eingeschlossenen Personen wurden mittels Niedrigdosis-Computertomographie (CT) in 8 Fällen $(4,3 \%)$ histologisch bestätigte Lungenkarzinome detektiert [4]. Dies zeigt, dass bei asbestexponierten Hochrisiko-Patienten mit einer relativ hohen Prävalenz von Lungentumoren gerechnet werden muss. Ob bei diesen Personen durch regelmäßige Screening-Untersuchungen mittels Niedrigdosis-CT die tumorspezifische Mortalität gesenkt werden kann, wurde bislang nicht untersucht.

Die im US-amerikanischen National Lung Screening Trial (NLST) bei Rauchern und Exrauchern im Alter von 55-74 Jahren erhobenen Daten deuten darauf hin, dass in diesem Kollektiv die lungenkarzinomspezifische Mortalität durch 3 jährliche Screening-Untersuchungen mittels Niedrigdosis-CT reduziert werden kann [5, 6] (Details und Ergebnisse NLST siehe Seite 6).

\section{Tabakprävention aus Sicht des Kinder- und Jugendpsychiaters}

Wiederholte Befragungen der Bundeszentrale für gesundheitliche Aufklärung (BZgA) zum Gebrauch von Drogen zeigen, dass der Zigarettenkonsum bei Kindern und Jugendlichen im Alter von 12-17 Jahren in letzter Zeit deutlich zurückgegangen ist. Während die Raucherquote in dieser Altersgruppe 1979 noch 38,5\% (männlich) bzw. 26,8\% (weiblich) betrug, belief sich der Raucheranteil 2010 auf 14,2\% bzw. 11,5\%. Der Anteil der Nieraucher, der 1979 33,4\% (männlich) bzw. 46,8\% (weiblich) betrug, stieg bis 2010 auf 66,5\% bzw. 69,7\%. Neben der Raucherquote insgesamt nahm bei Kindern und Jugendlichen seit 2003 auch der Anteil mit täglichem bzw. starkem ( $\geq 10$ Zigaretten täglich) Rauchkonsum leicht ab (Abb. 1) [7, 8].
Verhältnisprävention

- Erhöhung der Tabaksteuer (2002, 2003, 2004, 2005)

- Anhebung des legalen Bezugsalters auf 18 Jahre

- Modifikation und Reduktion von Zigarettenautomaten

- Implementierung rauchfreier Schulen

- Gesetzgebung zum Nichtraucherschutz

Verhaltensprävention mit Nichtraucherprogrammen

- «Rauchfrei»

- «Be Smart - Don't Start»

- «Just be smoke free»

- «Rauchfrei im Mai»

- «Nichtrauchen ist cool»

Neben einer Abnahme des Rauchkonsums zeichnete sich in den letzten Jahren zugleich ein leichter Anstieg des Erstkonsumalters ab. Nach Untersuchungen der vom Büro für Suchtprävention der Hamburgischen Landesstelle für Suchtfragen regelmäßig durchgeführten Schüler- und Lehrerbefragungen zum Umgang mit Suchtmitteln (Hamburger SCHULBUS) stieg bei 14- bis 18-jährigen Schüler/innen das Erstkonsumalter für Tabakrauchen zwischen 2004 und 2009 von 12,8 auf 13,2 Jahre.

\section{Verhältnispräventive Maßnahmen}

Grundlage der besonders im Vergleich zu anderen Präventionsprogrammen guten Ergebnisse der Tabakprävention bei Kindern und Jugendlichen sind verschiedene verhältnis- und verhaltenspräventive Maßnahmen (Kasten). Zu einem deutlichen Rückgang im Verkauf fabrikfertiger Zigaretten haben vor allem die Erhöhungen der Tabaksteuer in den Jahren 2002 bis 2005 geführt, wie eine in Deutschland bei Jugendlichen und Erwachsenen ( $\geq 15$ Jahre) durchgeführte Untersuchung zeigte (Abb. 2) [9].

\section{Verhaltenspräventive Maßnahmen}

Strikte Rauchverbote an Schulen sind am effektivsten, wenn sie mit möglichst interaktiven verhaltenspräventiven Maßnahmen kombiniert werden, die neben dem spezifischen Wissen zur Problematik des Rauchkonsums auch soziale und ProblemlöseKompetenzen fördern und den Jugendlichen Möglichkeiten aufzeigen, sich dem Gruppendruck zum Nikotinkonsum zu widersetzen. Die Ergebnisse des seit 1997/1998 durchgeführten Nichtraucherwettbewerbs «Be Smart - Don't Start», zu dem sich seit dem Schuljahr 2004/2005 in Deutschland jedes Jahr mehr als 10000 Schulklassen anmelden, zeigt einen deutlichen präventiven Effekt des Programms. Während in der Kontrollgruppe ohne verhaltenspräventive Intervention der Anteil der Raucher von der Baseline-Erhebung bis zur Katamnese 12-24 Monate nach der Eingangsuntersuchung von 14 auf 36\%, d.h. um 22\% zunahm, stieg der Raucheranteil in der Interventionsgruppe im gleichen Zeitraum nur um 16\% (von 12 auf 28\%) [10]. 


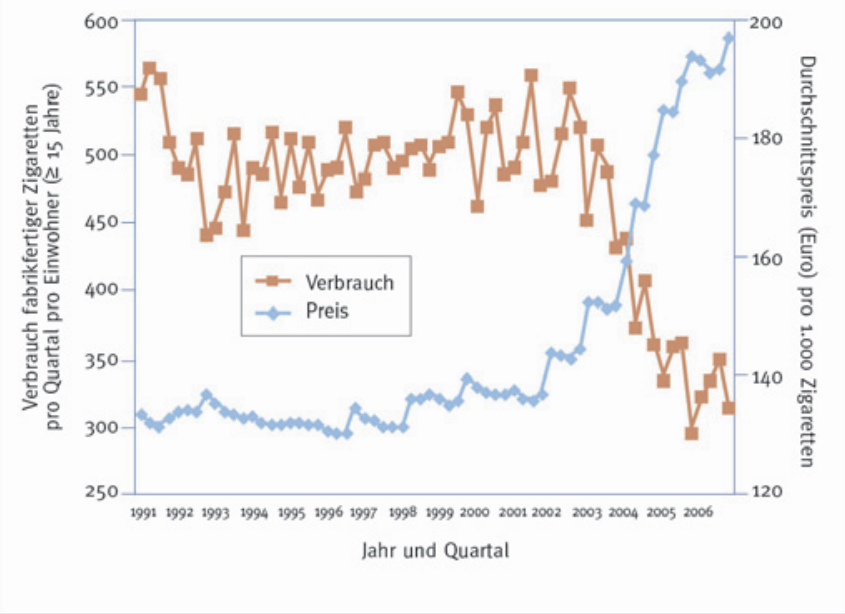

Abb. 2. Einfluss des Preises auf den Verbrauch fabrikfertiger Zigaretten (modifiziert nach [9]).

An der Aufklärungsveranstaltung «Nichtrauchen ist cool» haben am Universitätsklinikum Hamburg-Eppendorf (UKE) seit Mai 2005 etwa 40000 Schüler teilgenommen. Bei der von der Kinder- und Jugendpsychiatrie und Inneren Medizin konzipierten, einmal wöchentlich stattfindenden Veranstaltung erhalten Schüler der Klassen 5-7 didaktisch gut aufbereitete Informationen zum Thema Rauchen, anschließend wird ihnen die Videoaufzeichnung einer Bronchoskopie bei einem Tumorpatienten gezeigt. Besonders eindrucksvoll ist für die meisten Schüler die Begegnung mit einem betroffenen Patienten, der im direkten Gespräch von seiner Krankheit berichtet und Fragen der Schüler beantwortet.

Untersuchungen des Deutschen Zentrums für Suchtfragen des Kindes- und Jugendalters (DZSKJ), das das Programm wissenschaftlich evaluiert, zeigen präventive Effekte, die allerdings auf die Gruppe der Gymnasiasten begrenzt sind. In dieser Gruppe rauchten von den Schülern, die initial keine Zigarettenerfahrung hatten, 18 Monate später (d.h. etwa 15 Monate nach der Aufklärungsveranstaltung) in der Interventionsgruppe 4,6\%, in der Kontrollgruppe hatten $8 \%$ mit dem Rauchen angefangen.

Nach den Erfahrungen in Hamburg können von der Aufklärungskampagne nicht nur die teilnehmenden Schüler profitieren, sondern auch ihre rauchenden Eltern, die durch den Veranstaltungsbesuch ihrer Kinder motiviert werden, an einer Tabakentwöhnung teilzunehmen.

\section{Tabakentwöhnung aus Sicht des Internisten}

Die Empfehlungen der aktuellen S3-Leitlinie zur Prävention, Diagnostik, Therapie und Nachsorge des Lungenkarzinoms sehen vor:
- «Bei Patienten mit Lungenkarzinom sollte der Tabakkonsum regelmäßig hinterfragt und dokumentiert werden (Empfehlungsgrad A).»

- «Patienten mit Lungenkarzinom, die noch rauchen, sollten dazu motiviert werden, den Tabakkonsum zu beenden. Ihnen sollte eine qualifizierte Tabakentwöhnung angeboten werden (Empfehlungsgrad A)» [11].

Basis dieser Empfehlungen sind die Ergebnisse mehrerer Studien, die bei unterschiedlichen Kollektiven, darunter Patienten mit Lungenkarzinom, deutliche Vorteile eines Rauchstopps zeigten. Den Nutzen einer präoperativen Tabakentwöhnung, die etwa 6 Wochen vor einer elektiven Hüft- oder Knieoperation begonnen wurde, zeigte eine randomisierte klinische Studie mit 120 Patienten aus 3 Krankenhäusern in Dänemark. In der Gruppe mit Tabakentwöhnung war neben der Gesamtkomplikationsrate (18\% vs. $52 \%$; p = 0,0003) auch die Häufigkeit von wundbedingten Komplikationen (5\% vs. $31 \% ; \mathrm{p}=0,001)$ statistisch signifikant niedriger als in der Kontrollgruppe. Die Inzidenz kardiovaskulärer Komplikationen $(0 \%$ vs. $10 \% ; \mathrm{p}=0,08)$ und Zweitoperationen ( $4 \%$ vs. $15 \% ; p=0,07)$ war tendenziell niedriger, die Dauer des Krankenhausaufenthalts war in der Interventionsgruppe 2 Tage kürzer (11 vs. 13 Tage) [12].

Bestätigt wurden diese Ergebnisse für Patienten mit Lungenkarzinom durch die Auswertung der Daten von 7990 Patienten mit primärer Resektion eines Lungenkarzinoms, die in der Datenbank der US-amerikanischen Society of Thoracic Surgeons von 1999 bis 2007 erfasst wurden. Danach waren die Krankenhausmortalität und das Risiko für pulmonale Komplikationen nicht nur bei Nichtrauchern und bei Patienten, die mehr als 12 Monate vor der Operation das Rauchen aufgegeben hatten, niedriger als bei Rauchern; auch eine erst 1-12 Monate vor der Operation begonnene Raucherentwöhnung war mit einem niedrigeren Mortalitäts- und Komplikationsrisiko assoziiert [13].

Den negativen Einfluss des Zigarettenrauchens auf das Ansprechen auf eine platinhaltige Chemotherapie zeigten die Ergebnisse einer retrospektiven Studie, in der die Daten von 285 Patienten mit Lungenkarzinom, darunter 63\% aktive Raucher, ausgewertet wurden. Patienten ohne Therapieansprechen $(\mathrm{n}=191)$ hatten in der Vergangenheit statistisch signifikant stärker geraucht als Patienten mit objektivem Ansprechen $(67,8 \pm 35,1$ vs. 38,7 $\pm 27,1$ Packungsjahre; $p<0,001)$. Als wichtigster unabhängiger negativer Prädiktor für das Therapieansprechen wurde in einer multivariaten Analyse starker Tabakkonsum $\geq 40$ Packungsjahre identifiziert [14].

Vorteile des Rauchstopps bei einer Radiochemotherapie zeigte eine kanadische Studie mit 215 Rauchern mit kleinzelligem Lungenkarzinom im Stadium Limited Disease (LSCLC). Patienten, die während der Therapie auf das Rauchen verzichteten, lebten median 18,0 Monate, bei Fortsetzung des Zigarettenkonsums betrug das mediane Gesamtüberleben dagegen nur 13,6 Monate. Der Vergleich der 5-Jahres-Überlebensraten $(8,9 \%$ vs. $4,0 \%$; $p=0,0017)$ 
zeigte, dass die Tabakentwöhnung vor einer Radiochemotherapie das klinische Outcome auch langfristig verbesserte (Abb. 3) [15].

Nach Erfahrungen bei Patienten mit chronisch obstruktiven Lungenerkrankungen (COPD) [16] und bei langjähriger Begleitung von Tumorpatienten ist eine Tabakentwöhnung mit medikamentöser und verhaltenstherapeutischer Unterstützung auch bei Patienten mit Lungenkarzinom möglich, wobei die Bereitschaft zur Beendigung des Nikotinkonsums bei Besprechung der Erstdiagnose besonders groß ist. Im Beratungsgespräch können als Argumente für eine Tabakentwöhnung neben der Verbesserung des klinischen Outcomes auch Daten der Mayo-Klinik angeführt werden, wonach nicht rauchende Lungenkarzinom-Patienten (Nieraucher und Exraucher) eine bessere Tumor-spezifische Lebensqualität haben als Raucher [17].

Bei Lungenkarzinom-Patienten, die auf das Rauchen nicht verzichten wollen oder bei denen eine Tabakentwöhnung erfolglos ist, sollte der potenzielle Einfluss des Rauchens auf die Metabolisierung von Arzneistoffen berücksichtigt werden. Der orale epidermale Wachstumsfaktorrezeptor (EGFR)Inhibitor Erlotinib (Tarceva ${ }^{\circledR}$, Roche Pharma AG) wird bei Rauchern durch Induktion der Cytochrom-P450-Enzyme CYP1A1/1A2 stärker metabolisiert als bei Exrauchern oder

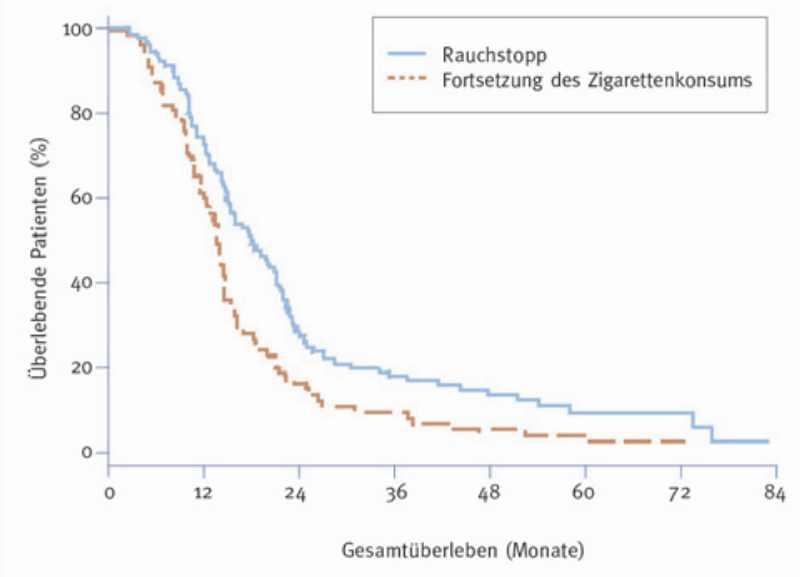

Abb. 3. Gesamtüberleben bei Patienten mit LSCLC bei einer Radiochemotherapie in Abhängigkeit vom Rauchverhalten (modifiziert nach [15]).
Nichtrauchern. In einer Phase-I/II-Studie bei chemotherapeutisch vorbehandelten Rauchern ( $\geq 10$ Zigaretten/Tag, $\geq 1$ Jahr) mit fortgeschrittenem nichtkleinzelligen Lungenkarzinom (NSCLC) betrug die maximal tolerable Dosis (MTD) von Erlotinib $300 \mathrm{mg} / \mathrm{Tag}$. Die bei dieser Dosierung gemessenen Plasmaspiegel lagen im selben Bereich wie die in früheren Studien bei Nichtrauchern unter täglich $150 \mathrm{mg}$ erreichten Konzentrationen. Auch die Inzidenz von Hauttoxizitäten und Diarrhö (Tab. 1) war bei Rauchern unter täglich 300 mg Erlotinib vergleichbar mit der bei Nichtrauchern, die mit täglich $150 \mathrm{mg}$ behandelt wurden [18-20].

\section{Lungenkrebs-Früherkennung mit radiologischen Verfahren}

Mit einer durchschnittlichen 5-Jahres-Überlebensrate von weniger als $15 \%$ haben Patienten mit NSCLC eine sehr ungünstige Prognose, wobei in Abhängigkeit vom Stadium bei Erstdiagnose allerdings große Unterschiede im Gesamtüberleben beobachtet werden. Während das 5-Jahres-Überleben bei Patienten mit nichtoperablem NSCLC im Stadium IIIB/ IV weniger als $5 \%$ beträgt, liegt die 5-Jahres-Überlebensrate im Tumorstadium IA bei mehr als $65 \%$. In frühen Tumorstadien (IA-IIB) werden derzeit allerdings nur etwa $25 \%$ der Patienten diagnostiziert.

Ziel der seit den 1970er-Jahren evaluierten radiologischen Verfahren zur Lungenkarzinom-Früherkennung ist es, durch vermehrte Detektion früher Tumorstadien die Lungenkarzinom-spezifische Mortalität zu senken. Mit regelmäßigen Röntgenthoraxaufnahmen konnten in klinischen Studien zwar mehr Lungenkarzinome erkannt werden, die Mortalität wurde aber nicht reduziert. Als Grund hierfür wurde die geringe Sensitivität der Thoraxübersichtsaufnahme für die Detektion früher Tumorstadien vermutet. Mittels konventioneller Thorax-CT lassen sich zwar auch kleine Rundherde detektieren, aufgrund der hohen Strahlenexposition eignet sich dieses Verfahren aber nicht zur routinemäßigen Früherkennung.

Die Bedeutung der Niedrigdosis-CT als Früherkennungsuntersuchung wurde in mehreren Machbarkeitsstudien mit ähnlichem Design (1-armig, keine Kontrollgruppe) und diagnostischem Algorithmus (Tumoren $>10 \mathrm{~mm}$ : primäre Biopsie; Tumoren $\leq 10 \mathrm{~mm}$ : Verlaufskontrolle, Biopsie erst bei Wachstum) untersucht und über insgesamt 18266 asympto-
Tab. 1. Inzidenz von Hauttoxizitäten und Diarrhö bei Behandlung mit Erlotinib

\begin{tabular}{lll}
\hline Unerwünschte Ereignisse & $\begin{array}{l}\text { Raucher, } \\
\text { Erlotinib } 300 \mathrm{mg} / \mathrm{Tag}\end{array}$ & $\begin{array}{l}\text { Nichtraucher, } \\
\text { Erlotinib } 150 \mathrm{mg} / \mathrm{Tag}\end{array}$ \\
\hline Hauttoxizitäten, \% & 67 & 88 \\
Diarrhö, \% & 50 & 55 \\
\hline
\end{tabular}

Modifiziert nach [18-20]. 
Tab. 2. NLST:

Lungenkarzinomspezifische Mortalität

\begin{tabular}{lllll}
\hline Studienarm & Personenjahre & $\begin{array}{l}\text { Lungenkarzinom- } \\
\text { Todesfälle }\end{array}$ & $\begin{array}{l}\text { Lungenkarzinom- } \\
\text { spezifische Mortalität } \\
\text { pro } 100 \text { 000 } \\
\text { Personenjahre }\end{array}$ & $\begin{array}{l}\text { Reduktion } \\
\text { Lungenkarzinom- } \\
\text { spezifische Mortalität, \% }\end{array}$ \\
\hline $\begin{array}{l}\text { Niedrigdosis-CT } \\
\text { Röntgen-Thorax }\end{array}$ & 144097,6 & 354 & 245,7 & 20,3 \\
\hline
\end{tabular}

Nach [6]. matische Personen mit erhöhtem Lungenkrebsrisiko berichtet. Bei 144 Patienten $(0,8 \%)$ wurde ein NSCLC diagnostiziert, das sich in $78 \%$ der Fälle im Stadium I befand und damit potenziell kurativ therapierbar war. Anschließend durchgeführte Folgeuntersuchungen zeigten eine Inzidenz von 0,4\%, wobei $84 \%$ der Tumoren dem Stadium I zugeordnet wurden. Der Anteil der Biopsien mit benignen Veränderungen (falsch positive Befunde) belief sich auf $30 \%$.

Ob durch Detektion früher Tumorstadien mittels Niedrigdosis-CT auch die Mortalität reduziert werden kann, lässt sich anhand dieser 1-armigen Screening-Studien nicht beurteilen, zumal der Einfluss systematischer Fehler, wie Lead-TimeBias, Length-Time-Bias und Overdiagnosis, in diesem Setting unbekannt ist. Zwar zeigte die International Early Lung Cancer Action Program (I-ELCAP)-Studie für Patienten im Tumorstadium I, die innerhalb des 1 . Monats nach der Diagnose operiert wurden, eine sehr hohe 10-Jahres-Überlebensrate von $92 \%$, aber auch in dieser Studie fehlte eine entsprechende Kontrollgruppe [21].

Eine mathematische Modellierung mit den Daten von 3246 in 3 Studien mittels CT gescreenten asymptomatischen Rauchern oder Exrauchern zeigte dagegen, dass durch das Screening zwar mehr Karzinome detektiert und reseziert wurden, die Zahl der Patienten mit fortgeschrittenen Tumorstadien und die Lungenkrebs-spezifische Mortalität aber unverändert waren [22].

\section{National Lung Screening Trial}

Die Wirksamkeit der Früherkennung mittels NiedrigdosisSpiral-CT oder Röntgen-Thorax in 2 Ebenen wurde im NLST prospektiv untersucht. Von 2002 bis 2004 wurden in 33 USamerikanischen Screening-Zentren mehr als 53000 aktive Raucher oder Exraucher im Alter von 55-74 Jahren mit einer Zigarettenanamnese von mindestens 30 Packungsjahren rekrutiert. Nach der basalen Untersuchung waren im jährlichen Abstand 2 weitere Kontrollen vorgesehen. Primärer Studienendpunkt war die Lungenkarzinom-spezifische Mortalität [5].

Als die Studie im Oktober 2010 vorzeitig beendet wurde, war im Niedrigdosis-CT-Arm die Inzidenz auffälliger Befunde deutlich höher $(24,2 \%$ vs. $6,9 \%)$ und die Lungenkarzinom-spezifische Mortalität wesentlich niedriger als in der Vergleichsgruppe, die mittels konventioneller Thoraxübersichtsaufnahmen untersucht worden war (Tab. 2) [6].
Basierend auf diesen Daten haben die Deutsche Gesellschaft für Pneumologie und Beatmungsmedizin und die Deutsche Röntgengesellschaft gemeinsam eine vorläufige Stellungnahme zur Lungenkrebsfrüherkennung erarbeitet [23], nach der eine native Niedrigdosis-CT des Thorax im Einzelfall gerechtfertigt sein kann, wenn bestimmte Standards einer Qualitätssicherung berücksichtigt werden. Danach ist vorgesehen, dass der Patient in einem ausführlichen Aufklärungsgespräch darauf hingewiesen wird, dass

- ein potenzieller Nutzen der Untersuchung nur für Raucher (mindestens 30 Packungsjahre) im Alter von 55-74 Jahren nachgewiesen wurde,

- tödlich verlaufende Lungenkarzinome am sichersten durch Aufgabe des Rauchens, gegebenenfalls mittels entsprechender Rauchentwöhnungsprogramme, vermieden werden können,

- bei Früherkennungsuntersuchungen asymptomatischer Personen unter anderem mit erforderlichen Kontrolluntersuchungen aufgrund gefundener Auffälligkeiten, unnötiger Beunruhigung, Biopsien und Operationen aufgrund falsch positiver Befunde und einer erhöhten Strahlenexposition $\mathrm{zu}$ rechnen ist.

\section{Stellenwert endoskopischer Verfahren bei der Lungenkrebs-Früherkennung}

In den zentralen Atemwegen lokalisierte prä- und frühmaligne Läsionen, die sich über mehrere Zwischenstufen zum invasiven Lungenkarzinom entwickeln, können mittels radiologischer Verfahren nicht erkannt werden und sind mittels Weißlicht-Bronchoskopie nur bei ausreichender Erfahrung mit hoher Sensitivität darstellbar. Eine Verbesserung der Sensitivität für frühe intraepitheliale Läsionen lässt sich durch zusätzliche Untersuchung mittels AutofluoreszenzBronchoskopie erreichen. Die zusätzliche Licht-induzierte Fluoreszenz-Endoskopie (LIFE) von im Weißlicht verdächtigen Läsionen verbesserte in der 1. publizierten Studie die Sensitivität bei intraepithelialen Neoplasien (moderate/ schwere Dysplasie und Carcinoma in situ (CIS)) um den Faktor 6,3; bei Einschluss invasiver Karzinome war die Sensitivität 2,7-mal größer als bei alleiniger Weißlicht-Bronchoskopie [24]. 
Tab. 3. Sensitivität, relative Sensitivität, Spezifität und relative Spezifität bronchoskopischer Verfahren bei der Detektion intraepithelialer Neoplasien

\begin{tabular}{|c|c|c|c|c|c|c|c|}
\hline & WLB & AFI & $\mathrm{WLB}+\mathrm{AFI}$ & NBI & $\mathrm{WLB}+\mathrm{NBI}$ & $\mathrm{AFI}+\mathrm{NBI}$ & $\mathrm{WLB}+\mathrm{NBI}+\mathrm{AFI}$ \\
\hline $\begin{array}{l}\text { Patienten mit Dysplasie } \\
\text { (moderat - schwer) und CIS, } \\
\text { die bronchoskopisch als } \\
\text { positiv identifiziert wurde } \\
(\mathrm{n}=17)\end{array}$ & 3 & 11 & 11 & 9 & 9 & 12 & 12 \\
\hline Sensitivität & 0,18 & 0,65 & 0,65 & 0,53 & 0,53 & 0,71 & 0,71 \\
\hline Relative Sensitivität & 1,0 & 3,7 & 3,7 & 3,0 & 3,0 & 4,0 & 4,0 \\
\hline $\begin{array}{l}\text { Patienten mit Metaplasie } \\
\text { und leichter Dysplasie, die } \\
\text { bronchoskopisch als negativ } \\
\text { identifiziert wurde }(\mathrm{n}=40)\end{array}$ & 35 & 16 & 14 & 36 & 31 & 16 & 14 \\
\hline Spezifität & 0,88 & 0,4 & 0,35 & 0,90 & 0,78 & 0,40 & 0,35 \\
\hline Relative Spezifität & 1,0 & 0,5 & 0,4 & 1,0 & 0,9 & 0,5 & 0,4 \\
\hline
\end{tabular}

$\mathrm{WLB}=$ Weißlicht-Video-Bronchoskopie, AFI = Autofluoreszenz-Bronchoskopie, NBI = Narrow-Band-Imaging-Bronchoskopie, CIS = Carcinoma in situ. Nach [25].

Zahlreiche Folgestudien bestätigten die verbesserte Sensitivität des Verfahrens für prä- und frühmaligne Läsionen, zeigten aber insgesamt eine relativ geringe Spezifität, da verschiedene nichtmaligne Gewebe, beispielsweise Narben und Entzündungen, ähnliche Fluoreszenz-Phänomene auslösen. Bei Einsatz der Autofluoreszenz-Bronchoskopie muss daher mit einer erhöhten Zahl an erforderlichen Biopsien gerechnet werden.

Eine deutlich höhere Spezifität als die AutofluoreszenzBronchoskopie hat die Narrow-Band-Imaging (NBI)-Bronchoskopie, bei der mittels verschiedenfarbiger, schmaler Lichtbänder unterschiedliche Ebenen der Bronchialmukosa untersucht werden können. Die Leistungsfähigkeit des neuen Verfahrens allein sowie in Kombination mit der Weißlicht-Video-Bronchoskopie (WLB) und Autofluoreszenz-Bronchoskopie (AFI) wurde in einer Studie mit 62 Personen untersucht, die zur Früherkennung oder Nachkontrolle bronchoskopiert wurden. Nach Ausschluss von 5 Patienten mit nachgewiesenem invasiven Lungenkarzinom betrug die Prävalenz intraepithelialer Neoplasien $30 \%$. Bei einer Sensitivität der WLB von 0,18 wurde die relative Sensitivität der NBI und AFI mit 3,0 (p = $0,03)$ und 3,7 ( $\mathrm{p}=0,005)$ berechnet. Dabei war die Spezifität der NBI ebenso hoch wie die der WLB (Tab. 3) [25].

Mit der optischen Kohärenztomographie (OCT) können nichtinvasiv auch tiefere Schichten der Bronchialwand mit hoher Auflösung dargestellt und frühe Läsionen wie in einem histologischen Präparat detektiert werden. Mittels DopplerOCT lässt sich auch die Vaskularisierung der betreffenden Bereiche darstellen.

Obwohl mit den neuen Farbverfahren mehr frühe Veränderungen nachweisbar sind, haben sie für die routinemäßige Früherkennung derzeit noch keine Bedeutung. Dies liegt unter anderem daran, dass es bisher keine Selektionskriterien gibt, nach denen die von einer endoskopischen Früherken- nung besonders profitierenden Personen identifiziert werden können. Außerdem fehlen Marker, die Vorhersagen zur Entwicklung endoskopisch detektierter Läsionen im Einzelfall ermöglichen. Aus einer Metaplasie kann zwar eine Dysplasie und CIS entstehen, die Veränderung kann sich aber auch wieder vollständig zurückbilden. Bei Detektion einer Dysplasie oder eines CIS ist ebenfalls unklar, ob sich im Einzelfall daraus ein invasives Lungenkarzinom entwickelt.

\section{Zytologische Verfahren zur Früherkennung und Diagnosesicherung von Lungentumoren}

Studien zur Bedeutung der konventionellen Sputumzytologie im Rahmen von Screening-Untersuchungen konnten bisher weder für die konventionelle Sputumzytologie allein noch in Kombination mit radiologischen Verfahren einen Überlebensvorteil zeigen. Da auch durch Metaanalysen kein Überlebenszeitgewinn belegbar war, sprachen sich die an der interdisziplinären S3-Leitlinie zur Prävention, Diagnostik, Therapie und Nachsorge des Lungenkarzinoms beteiligten Experten aufgrund der derzeitigen Datenlage gegen ein Screening mittels Sputumzytologie aus (Empfehlungsgrad A) [11].

\section{Zytologische Diagnosesicherung}

Durch die Nachweismöglichkeit maligner (benigner) Tumorzellen in sehr kleinen Proben kann die Zytologie bei sehr schneller Aufarbeitung und rascher Effektivitätsbeurteilung in der Hand des Erfahrenen einen wichtigen Beitrag zur frühen Diagnostik von Lungentumoren leisten. In diesem Rahmen kann nach den Empfehlungen der S3-Leitlinie «die zytologische Diagnose eines NSCLC (via Bronchoskopie, Nadelpunktionsverfahren oder Sputum) mit hoher Zuverlässigkeit für das therapeutische Management akzeptiert werden (Emp- 


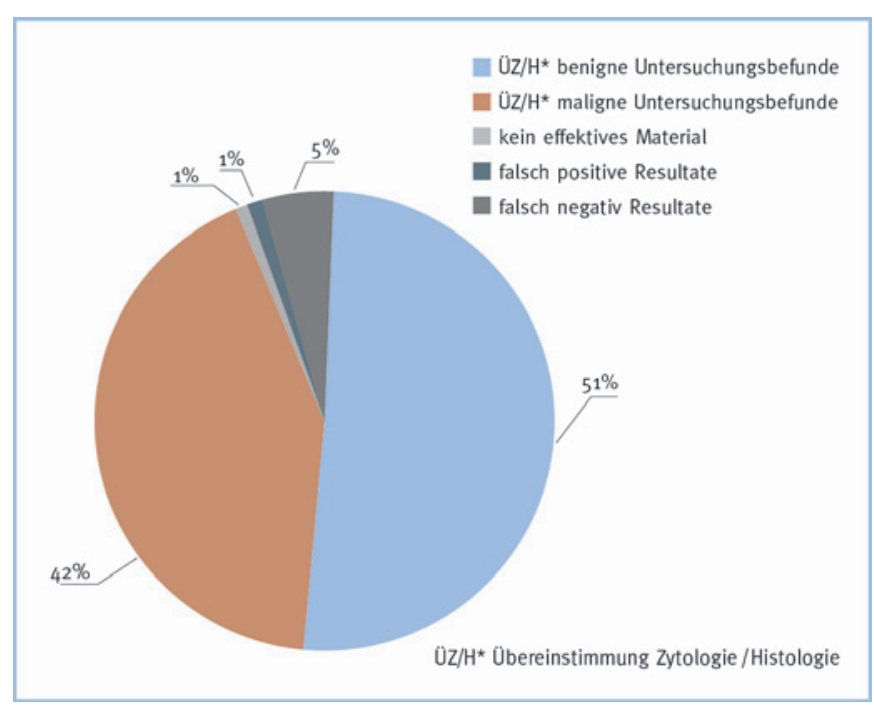

Abb. 4. Ergebnisse der intraoperativen Schnellzytologie am Krankenhaus Coswig.

fehlungsgrad A)». Bei der zytologischen Diagnose eines kleinzelligen Lungenkarzinoms (SCLC) sollte bei Diskrepanz zum klinischen Erscheinungsbild oder Verlauf ein bioptisches Verfahren zur histologischen/immunhistochemischen Diagnosesicherung durchgeführt werden (Empfehlungsgrad B). In hoch palliativen Krankheitssituationen steht die Zytologie als ausreichend sichere Methode zum Tumornachweis via Feinnadelpunktion zur Verfügung.

Grundlage der Empfehlungen waren unter anderem die Ergebnisse einer systematischen Analyse der bis 2001 publizierten Studien, in denen bei Patienten mit Verdacht auf Lungenkarzinom die Genauigkeit der zytologischen Diagnostik mit der Histologie verglichen wurde. Die für die Sputumzytologie auswertbaren Daten von 16 Studien mit mehr als 28000 Patienten zeigten eine gepoolte Spezifität von 99\%; die gepoolte Sensitivität betrug $66 \%$, wobei zentrale Läsionen mit höherer Sensitivität detektiert wurden als periphere (71\% vs. 49\%) [26].

Die Sensitivität der flexiblen Bronchoskopie war bei endobronchialen Befunden am größten, wenn die Entnahme mittels endobronchialer Biopsie erfolgte (74\%), bei der Entnahme mittels Bürste betrug sie 59\% und bei der Spülung $48 \%$. Bei Kombination aller Verfahren erhöhte sich die Sensitivität auf $88 \%$. Bei peripheren Läsionen war die Sensitivität schlechter (Bürste 52\%, transbronchiale Biopsie 46\%, Spülung $43 \%$ ), auch bei Kombination aller Methoden betrug die diagnostische Ausbeute nur 69\%. Die Auswertung von 8 Studien mit 341 Patienten, in denen die Sensitivität in Abhängigkeit von der Größe der Läsion untersucht wurde, zeigte bei kleinen Läsionen $(<2 \mathrm{~cm}$ ) eine Sensitivität von nur 33\% (vs. $62 \%$ bei größeren Läsionen). Durch transthorakale Nadelbiopsie konnte die Sensitivität der zytologischen Diagnostik auf $90 \%$ erhöht werden, wobei Läsionen $<2 \mathrm{~cm}$ aber auch hier mit geringerer Sensitivität detektierbar waren. Die Sensitivität für die Unterscheidung von NSCLC und SCLC betrug 98\%.
Auswertungen der im Krankenhaus Coswig 2006/2007 untersuchten Materialien $(\mathrm{n}=1141)$ zeigten eine hohe Übereinstimmung der intraoperativen Schnellzytologie und endgültigen Histologie (Abb. 4).

\section{Pulmonaler Rundherd: Thoraxchirurgische versus internistische Strategien}

Solitäre pulmonale Rundherde sind definiert als rundliche Läsionen mit einem Durchmesser von höchstens $3 \mathrm{~cm}$, die bei fehlenden Umgebungsauffälligkeiten (z.B. Atelektase) vollständig von Lungenparenchym umgeben sind. Die meisten pulmonalen Rundherde werden bisher bei Röntgenübersichtsaufnahmen als Zufallsbefund gesehen. Eine Zunahme der Prävalenz und Inzidenz ist zu erwarten, wenn Patienten mit erhöhtem Lungenkarzinom-Risiko im Rahmen der Früherkennung regelmäßig mittels Niedrigdosis-CT untersucht werden.

Die wichtigsten Kriterien bei der diagnostischen Abklärung pulmonaler Rundherde sind

- die Malignomwahrscheinlichkeit,

- das Operationsrisiko,

- der Wille des Patienten.

\section{Invasive Verfahren}

Nach dem Diagnosealgorithmus der Thoraxklinik am Universitätsklinikum Heidelberg ist - unter Berücksichtigung des Patientenwunsches - folgende Vorgehensweise üblich: Bei pulmonalen Rundherden $<5 \mathrm{~mm}$ erfolgt eine CT-Kontrolle nach 12 und 24 Monaten, Rundherde zwischen 5 und $10 \mathrm{~mm}$ werden nach 3, 6, 12 und 24 Monaten kontrolliert. Bei Progression wird bei funktioneller Operabilität die Indikation zur invasiven Abklärung mittels videoassistierter Thorakoskopie (VATS) gestellt. Bei größeren Rundherden (> $10 \mathrm{~mm}$ ) wird unter Abwägen der oben genannten 3 Kriterien direkt die invasive Abklärung empfohlen.

Verglichen mit nichtinvasiven Verfahren zur Rundherdabklärung, wie die transbronchiale Biopsie (BRSK-TBB) und die CT-gesteuerte transthorakale Biopsie (CT-TTB), zeichnet sich die VATS-Resektion bei peripheren Rundherden $\leq 2 \mathrm{~cm}$ durch die diagnostische Ausbeute von 100\% aus (Tab. 4). Psychische Belastungen infolge der bei unklarem BiopsieBefund erforderlichen Folgeuntersuchungen können bei VATS-Rundherd-Resektion vermieden werden.

\section{Bronchoskopische Verfahren}

Die interdisziplinäre S3-Leitlinie Lungenkarzinom empfiehlt bei pulmonalen Rundherden eine chirurgische Abklärung. Unter Umständen können aber auch bronchoskopische Verfahren zum Einsatz kommen. Die diagnostische Ausbeute bei flexibler Biopsie korrelierte in einer retrospektiven Studie mit 177 Patienten vor allem mit der Größe des Rundherds. Während Läsionen $\leq 2 \mathrm{~cm}$ nur in $23 \%$ der Fälle abgeklärt werden 
Tab. 4. Verfahren zur diagnostischen Abklärung peripherer pulmonaler Rundherde $\leq 2 \mathrm{~cm}$

\begin{tabular}{llll}
\hline & BRSK-TBB & CT-TTB & VATS \\
\hline Schnitte & nein & $0,5 \mathrm{~cm}$ & $1,0 \mathrm{~cm}+1,5 \mathrm{~cm}$ \\
Narkose & lokal (allgemein) & lokal & Doppellumen-Intubation \\
Dauer & $20-30 \mathrm{~min}$ & $20-30 \mathrm{~min}$ & $20-30 \mathrm{~min}$ \\
Drainagehäufigkeit & $10 \%$ & $5 \%$ & $100 \%$ \\
Stationärer Aufenthalt & 1 Tag & 1 Tag & $2-3$ Tage \\
Röntgenstrahlen-Belastung & ja & ja & nein \\
Diagnostische Ausbeute & $30-80 \%$ & $60-80 \%$ & $100 \%$ \\
\hline
\end{tabular}

BRSK-TBB = Transbronchiale Biopsie, CT-TTB $=$ CT-gesteuerte transthorakale Biopsie, VATS = videoassistierte Thorakoskopie.
Tab. 5. Diagnostische Ausbeute bei flexibler Bronchoskopie in Abhängigkeit von der Größe und Dignität der Läsion

\begin{tabular}{llrr}
\hline Größe der Läsion & Maligne Veränderung & Benigne Veränderung & Insgesamt \\
\hline$\leq 2 \mathrm{~cm}$ & $4 / 16(25 \%)$ & $3 / 14(21 \%)$ & $7 / 30(23 \%)$ \\
$2,1-2,5 \mathrm{~cm}$ & $7 / 18(39 \%)$ & $1 / 2(50 \%)$ & $8 / 20(40 \%)$ \\
$2,6-4 \mathrm{~cm}$ & $39 / 61(64 \%)$ & $4 / 8(50 \%)$ & $43 / 69(62 \%)$ \\
$>4 \mathrm{~cm}$ & $47 / 56(84 \%)$ & $1 / 2(50 \%)$ & $48 / 58(83 \%)$ \\
Alle Läsionen & $97 / 151(64 \%)$ & $9 / 26(35 \%)$ & $106 / 177(60 \%)$ \\
\hline
\end{tabular}

Modifiziert nach [27].

konnten, betrug die diagnostische Ausbeute bei Läsionen $>4 \mathrm{~cm} 83 \%$ (Tab. 5). Als weitere Determinanten für die diagnostische Ausbeute wurden der Abstand vom Hilus, das betroffene Segment und die Dignität identifiziert [27].

Mittels CT-gesteuerter Feinnadelpunktion kann auch bei kleineren Rundherden eine größere diagnostische Ausbeute erreicht werden. Besonders bei kleineren Läsionen $\leq 2 \mathrm{~cm}$ muss allerdings vermehrt mit pulmonalen Komplikationen, vor allem Pneumothorax und Blutungen gerechnet werden. Die retrospektive Analyse von 660 Biopsien zeigte eine Pneumothoraxrate von $23 \%$, wobei Patienten mit einer Läsion $\leq 2 \mathrm{~cm}$ deutlich häufiger betroffen waren als solche mit Läsionen $\geq 4,1 \mathrm{~cm}$ (33 vs. $15 \%$ ). Auch Hämoptysen, die bei $4 \%$ der Patienten auftraten, wurden häufiger bei Patienten mit kleinen Rundherden gesehen [28].

Patienten mit kleineren Läsionen können möglicherweise vom Einsatz eines elektromagnetischen Navigationssystems profitieren. In einer prospektiven Studie mit 60 Patienten, die verdächtige Läsionen mit einer durchschnittlichen Größe von $22,8 \pm 12,6 \mathrm{~mm}$ und/oder vergrößerte Lymphknoten $(28,1 \pm$ $12,8 \mathrm{~mm}$ ) aufwiesen, betrug die diagnostische Ausbeute 74\% (Rundherde) bzw. 100\% (Lymphknoten). Die Pneumothoraxrate belief sich auf 3,5\% [29]. Bei 40 konsekutiven Patienten mit ausgeprägten Komorbiditäten lag die diagnostische Ausbeute zwischen 43,7\% (Läsionen > $10 \leq 20 \mathrm{~mm}$ ) und $71,4 \%$ (Läsionen $>20 \leq 30 \mathrm{~mm}$ ) [30]. Ob die Bronchoskopie mit elektromagnetischer Navigation der konventionellen Durchleuchtung generell oder bei kleineren Läsionen tatsächlich überlegen ist, ist aufgrund fehlender Vergleichsuntersuchungen derzeit allerdings noch unklar.

\section{Disclosure Statement}

Die Autoren waren Referenten beim «Dresdner Interdisziplinäres Gespräch», das von der Roche Pharma AG unterstützt wurde.

\section{Literatur}

1 Hauptverband der gewerblichen Berufsgenossenschaften (HVBG): Beruflich verursachte Krebserkrankungen. Dokumentation des Berufskrankheiten-Geschehens in Deutschland. Eine Darstellung der im Zeitraum 1978 bis 2003 anerkannten Berufskrankheiten, ed 8. Sankt Augustin, HVBG, 2005.

2 Nowak D: Berufskrebs - Überlegungen zur arbeitsmedizinischen Risikoabschätzung. Arbeitsmed Sozialmed Umweltmed 1998;33:334-343.
3 De Matteis S, Consonni D, Bertazzi PA: Exposure to occupational carcinogens and lung cancer risk. Evolution of epidemiological estimates of attributable fraction. Acta Biomed 2008;79(suppl 1):34-42.

4 Das M, Mühlenbruch G, Mahnken AH, et al.: Asbestos Surveillance Program Aachen (ASPA): initial results from baseline screening for lung cancer in asbestos-exposed high-risk individuals using low-dose multidetector-row CT. Eur Radiol 2007; 17:1193-1199.
5 Aberle DR, Berg CD, Black WC, et al.: The National Lung Screening Trial: overview and study design. Radiology 2011;258:243-253.

6 www.cancer.gov/images/dsmb-nlst.pdf

7 Bundeszentrale für gesundheitliche Aufklärung (BZgA): Die Drogenaffinität Jugendlicher in der Bundesrepublik Deutschland 2008. Verbreitung des Tabakkonsums bei Jugendlichen und jungen Erwachsenen. Köln, BZgA, 2009. 
8 http://fdr-online.info/media/pdf-Dateien/Tagungsbeitraege/03_Orth.pdf.

$\checkmark$ Hanewinkel R, Radden C, Rosenkranz T: Price increase causes fewer sales of factory-made cigarettes and higher sales of cheaper loose tobacco in Germany. Health Econ 2008;6:683-693.

10 Hanewinkel R: «Be Smart - Don’t Start». Ergebnisse des Nichtraucherwettbewerbs in Deutschland 1997-2007. Gesundheitswesen 2007;69:38-44.

11 Goeckenjan G, Sitter H, Thomas M, et al.: Prävention, Diagnostik, Therapie und Nachsorge des Lungenkarzinoms. Pneumologie 2010;64(suppl 2): S23-S155.

-12 Møller AM, Villebro N, Pedersen T, et al.: Effect of preoperative smoking intervention on postoperative complications: a randomised clinical trial. Lancet 2002;359:114-117.

13 Mason DP, Subramanian S, Nowicki ER, et al.: Impact of smoking cessation before resection of lung cancer: a Society of Thoracic Surgeons General Thoracic Surgery Database study. Ann Thorac Surg 2009;88:362-370.

14 Duarte RL, Luiz RR, Paschoal ME: The cigarette burden (measured by the number of pack-years smoked) negatively impacts the response rate to platinum-based chemotherapy in lung cancer patients. Lung Cancer 2008;61:244-254.

15 Videtic GM, Stitt LW, Dar AR, et al.: Continued cigarette smoking by patients receiving concurrent chemoradiotherapy for limited-stage small-cell lung cancer is associated with decreased survival. $\mathrm{J}$ Clin Oncol 2003;21:1544-1549.
16 Andreas S, Batra A, Behr J, et al.: Tabakentwöhnung bei COPD. S3-Leitlinie herausgegeben von der Deutschen Gesellschaft für Pneumologie und Beatmungsmedizin. Pneumologie 2008;62:255-272.

17 Garces YI, Yang P, Parkinson J, et al.: The relationship between cigarette smoking and quality of life after lung cancer diagnosis. Chest 2004;126: 1733-1741.

18 Hughes AN, O'Brien MER, Petty WJ, et al.: Overcoming CYP1A1/1A2 mediated induction of metabolism by escalating erlotinib dose in current smokers. J Clin Oncol 2009;27:1220-1226.

19 Clark GM, Zborowski DM, Santabarbara P, et al.: Smoking history and epidermal growth factor receptor as predictors of survival benefit from erlotinib for patients with non-small-cell lung cancer in the National Institute of Canada Clinical Trials Group study BR.21. Clin Lung Cancer 2006;7:389-394.

20 Shepherd FA, Pereira JR, Ciuleanu T, et al.: Erlotinib in previously treated non-small-cell lung cancer. N Engl J Med 2005;353:123-132.

21 Henschke CI, Yankelevitz DF, Libby DM, et al.: Survival of patients with stage I lung cancer detected on CT screening. N Engl J Med 2006; 355:1763-1771.

22 Bach PB, Jett JR, Pastorino U, et al.: Computed tomography screening and lung cancer outcomes. JAMA 2007;297:953-961.
23 Vogelmeier C, Worth H, Pfeifer M, et al.: Gemeinsame Stellungnahme der Deutschen Gesellschaft für Pneumologie und Beatmungsmedizin und der Deutschen Röntgengesellschaft zur Lungenkrebsfrüherkennung mit Niedrigdosis-CT. Pneumologie 2011;65:5-6.

24 Lam S, Kennedy T, Unger M, et al.: Localization of bronchial intraepithelial neoplastic lesions by fluorescence bronchoscopy. Chest 1998;113:696-702.

25 Herth FJ, Eberhardt R, Anantham D, et al.: Narrow-band imaging bronchoscopy increases the specificity of bronchoscopic early lung cancer detection. J Thorac Oncol 2009;4:1060-1065.

26 Schreiber G, McCrory DC: Performance characteristics of different modalities for diagnosis of suspected lung cancer: summary of published evidence. Chest 2003;123(suppl 1):115S-128S.

27 Baaklini WA, Reinoso MA, Gorin AB, et al.: Diagnostic yield of fiberoptic bronchoscopy in evaluating solitary pulmonary nodules. Chest 2000; 117:1049-1054.

28 Yeow KM, Su IH, Pan KT, et al.: Risk factors of pneumothorax and bleeding: multivariate analysis of 660 CT-guided coaxial cutting needle lung biopsies. Chest 2004;126:748-754.

29 Gildea TR, Mazzone PJ, Karnak D, et al.: Electromagnetic navigation diagnostic bronchoscopy: a prospective study. Am J Respir Crit Care Med 2006;174:982-989.

-30 Makris D, Scherpereel A, Leroy S, et al.: Electromagnetic navigation diagnostic bronchoscopy for small peripheral lung lesions. Eur Respir J 2007; 29:1187-1192. 